\section{Pediatric laparoscopy and adaptive oxygenation and hemodynamic changes}

Gloria Pelizzo, 1 Veronica Carlini, 2

Giulio Iacob,2 Noemi Pasqua,2

Giuseppe Maggio, ${ }^{3}$ Marco Brunero, 2

Simonetta Mencherini, ${ }^{3}$

Annalisa De Silvestri, ${ }^{4}$

Valeria Calcaterra,, $\mathbf{5 , 6}$

1Pediatric Surgery Unit, Children's

Hospital, Istituto Mediterraneo di

Eccellenza Pediatrica, Palermo;

${ }^{2}$ Pediatric Surgery Unit, Fondazione

IRCCS Policlinico San Matteo Pavia;

${ }^{3}$ Anesthesiology and Intensive Care

Unit, Fondazione IRCCS Policlinico San

Matteo, Pavia; ${ }^{4}$ Biometry and Clinical

Epidemiology, Scientific Direction,

Fondazione IRCCS Policlinico San

Matteo, Pavia; 5Pediatric Unit,

Department of Maternal and Child

Health Fondazione IRCCS Policlinico

San Matteo Pavia; 6Department of

Internal Medicine and Therapeutics,

University of Pavia, Italy

\section{Abstract}

Adaptive changes in oxygenation and hemodynamics are evaluated during pediatric laparoscopy. The children underwent laparoscopy (LAP Group, $\mathrm{n}=20$ ) or open surgery (Open Group, $\mathrm{n}=10$ ). Regional cerebral $\left(\mathrm{rScO}_{2}\right)$ and peripheral oxygen saturation $\left(\mathrm{SpO}_{2}\right)$, heart rate (HR), diastolic (DP) and systolic pressure (SP) were monitored at different intervals: basal (T0); anesthesia induction (T1); $\mathrm{CO}_{2} \mathrm{PP}$ insufflation (T2); surgery (T3); $\mathrm{CO}_{2} \mathrm{PP}$ cessation (T4); before extubation (T5). At T1, in both the LAP and Open groups significant changes in $\mathrm{rScO}_{2}$, DP and SP were recorded compared with T0; a decrease in $\mathrm{SatO}_{2}$ was also observed at T5. In the LAP group, at T2, changes in $\mathrm{HR}$ related to $\mathrm{CO}_{2} \mathrm{PP}$ pressure and in DP and SP related to IAP were noted; at T4, a SP change associated with $\mathrm{CO}_{2} \mathrm{PP}$ desufflation was recorded. Open group, at $\mathrm{T} 3$ and $\mathrm{T} 5$ showed lower $\mathrm{rScO}_{2}$ values compared with T1. Pneuperitoneum and anesthesia are influent to induce hemodynamics changes during laparoscopy.

\section{Introduction}

The laparoscopic surgical technique in children and infants is still undergoing development and refinement. To date, its effects on systemic and cerebral oxygenation have not been completely investigated ${ }^{1-3}$ and the exact effects of surgical maneuvers in combination with conventional anesthesiological procedures in hemodynamic regulation are still debated. Hemodynamic alterations, associated with abdominal laparoscopy are mainly caused by the increased intra-abdominal pressure (IAP), brought on by pneumoperitoneum (PP) creation. A decrease in venous return secondary to inferior vena cava compression and the increase in central venous pressure and arterial blood pressure, in the absence of heart rate (HR) changes, seem to be the main adaptive responses. ${ }^{4-6} \mathrm{~A} 10 \%$ to $30 \%$ decrease in cardiac output with severe pathophysiologic modifications has also been reported in most studies, ${ }^{7-9}$ underscoring the need for dedicated anesthesiological support in pediatric laparoscopy, especially in infants. Pathophysiological hemodynamic alterations during laparoscopic procedures in children have not been fully investigated. ${ }^{10-15}$ Therefore, in small children and in surgical procedures of long duration, the standardization of mini invasive intraoperative assessment requires close anesthesiological monitoring to prevent adverse hemodynamic outcomes. ${ }^{16,17}$

The anesthesiologist should have a deep understanding of the consequences associated with PP creation; and it is critical that the anesthesiologist be prepared to detect and address possible alterations that may occur during laparoscopic interventions.

In the present study, we evaluated whether adaptive changes in cerebral and systemic oxygenation and in hemodynamics are intraoperatively induced by IAP increases and PP-associated $\mathrm{CO} 2$ changes. The contribution of knowledge on the real impact of anesthesia on hemodynamic regulation during pediatric laparoscopic procedures was also considered.

\section{Materials and Methods}

\section{Patients}

The initial inclusion criteria for recruitment in the study were as follows: thirty children $(21 \mathrm{M} / 9 \mathrm{~F})$, aged 1 to 18 years (mean age $8.1 \pm 5.1 \mathrm{yr}$ ), scheduled for elective abdominal surgical procedures for congenital unilateral inguinal hernia repair. The treatment group (LAP Group: 20 children, $12 \mathrm{M} / 8 \mathrm{~F}$, mean age $9.6 \pm 5.1$ ) underwent laparoscopic surgery, while the control group underwent traditional open surgery (Open Group: 10 children, 9M/1F, mean age $5.1 \pm 4.1)$.
Correspondence: Gloria Pelizzo, Pediatric Surgery Unit, Children Hospital, Via dei Benedettini n.1, 90134 Palermo, Italy.

Tel.: +39.091.6666007 - Fax: +39.091.6666006. E-mail: gloriapelizzo@gmail.com

Key words: laparoscopy; children; hemodinamics; changes.

Acknowledgments: the authors would thank Dr. L. Kelly for English revision of the manuscript.

Contributions: GP performed surgical treatment, drafted and critically revised the manuscript; $\mathrm{VeC}$ performed surgical treatment, collected the patient data, drafted the manuscript; GI collected the patient data; NP collected the patient data; GM performed anesthesiological support, collected the patient data and drafted the manuscript; MB performed surgical treatment; SM performed anesthesiological support, collected the patient data; ADS performed statistical analysis; $\mathrm{VaC}$ revised the literature, drafted and critically revised the manuscript.

Conflict of interest: the authors declare no potential conflict of interest.

Received for publication: 7 May 2017.

Revision received: 2 June 2017.

Accepted for publication: 3 June 2017.

This work is licensed under a Creative Commons Attribution NonCommercial 4.0 License (CC BY-NC 4.0).

(C) Copyright G. Pelizzo et al., 2017

Licensee PAGEPress, Italy

Pediatric Reports 2017; 9:7214

doi:10.4081/pr.2017.7214

Surgery was performed by an experienced surgeon, under general endotracheal anesthesia. To critically analyze and synthesize current evidence, we performed an analysis of the completed results from both groups. Patients were consecutively recruited between 1 February 2016 and 31 June 2016, at the Pediatric Surgery Unit of the Fondazione IRCCS Policlinico San Matteo, Pavia, Italy. Written consent was obtained from the parents of the children before the scheduled surgical procedure. The study was performed according to the Declaration of Helsinki and with the approval of the Institutional Review Board.

\section{Surgery}

All surgeries were performed in the same operating theater with a stable temperature of $22 \pm 1^{\circ} \mathrm{C}$. Patients were placed in the supine position on a heated operating table $\left(36 \pm 1^{\circ} \mathrm{C}\right)$. Laparoscopic treatment was accomplished, following a standard proto- 
col, via the trans-abdominal approach using one $3 \mathrm{~mm}$ telescope and two, $3 \mathrm{~mm}$ or 2 $\mathrm{mm}$, surgical instruments, placed into the lower abdomen. The pneumoperitoneum (PP) was created with a $3 \mathrm{~mm}$ infra-umbilical camera-trocar placed via an open approach. The $\mathrm{PP} \mathrm{CO}_{2}$ pressure, which ranged from 8 to $12 \mathrm{mmHg}(8 \mathrm{mmHg}$ in children weighing $<15 \mathrm{~kg} ; 10 \mathrm{mmHg}: 15-40$ $\mathrm{kg} ; 12 \mathrm{mmHg}$ : $>40 \mathrm{~kg}$ ), was achieved with a $\mathrm{CO}_{2}$ insufflation flow rate of $1 \mathrm{~L} / \mathrm{min}$.

Patients included in the control group underwent a traditional open-surgery procedure. All interventions were performed on a day hospital basis and patients were followed for one week.

\section{Data acquisition}

Intraoperative transcranial near-infrared spectroscopy (NIRS) was used to assess regional cerebral oxygen saturation $\left(\mathrm{rScO}_{2}\right)$, pulse oximetry was used to measure peripheral oxygen saturation using $\left(\mathrm{SpO}_{2}\right)$; and $\mathrm{HR}$, diastolic (DP), systolic pressure (SP) and end-tidal $\mathrm{CO}_{2}$ were monitored continuously during the entire procedure.

Changes in $\mathrm{rScO}_{2}$ were measured using a near-infrared spectrometer. Prior to anesthesia induction, a transducer was placed on the frontal side of the child's head and attached with an elastic bandage to prevent displacement. The oximeter sensor was positioned on the middle finger of the left hand. HR was recorded during scanning also using pulse oximetry for heart timing and an index of pulse amplitude.

Indirect data on IAP during surgery were collected via dynamic urethral pressure measurements with a high-resolution manometry system and recorded with Medical Measurement Systems ${ }^{\circledR}$ (Enschede, the Netherlands).

The following parameters were analyzed, every minute for five minutes (mean values were used for the statistical analysis), at five intervals: basal (T0) in LAP and Open groups; induction of anesthesia (T1) in LAP and Open groups; $\mathrm{CO}_{2} \mathrm{PP}$ insufflation (T2) in the LAP group; surgery (T3) in LAP and Open groups; cessation of $\mathrm{CO}_{2} \mathrm{PP}$ (T4) in the LAP group; before extubation (T5) in LAP and Open groups.

The operative times were recorded, including data acquisition and anesthesia duration (interval from beginning of induction to cessation of sevoflurane inhalation). The anesthesiologists were not blinded to the data readings to prevent intraoperative alterations in the children.

\section{Anesthesia protocol}

All children were in good physical con- dition (ASA, American Society of Anesthesiologists, class 1) and received standard anesthesia. Induction was performed endovenously (e.v.) with propofol (2-4 $\mathrm{mg} / \mathrm{Kg})$ and fentanyl $(1 \mu \mathrm{g} / \mathrm{Kg})$; and for muscle relaxation, cisatracurium 0.1 $\mathrm{mg} / \mathrm{Kg}$ was given e.v.

After tracheal tube positioning, patients underwent volume controlled mechanical ventilation with a tidal volume of $8 \mathrm{~mL} / \mathrm{Kg}$, the respiratory rate adjusted to achieve an end-tidal $\mathrm{CO}_{2}$ of $32-37 \mathrm{mmHg}$, an I:E ratio of 1:2 (avoiding dynamic hyperinflaction) with a low-flow breathing system and an inspired mixture of air and oxygen (fresh gas flow of $41 \mathrm{~min}^{-1}$ with $40 \% \mathrm{FiO}_{2}$ during anesthesia). Anesthesia was maintained via administration of Sevoflurane gas (0.9 to 1.3 MAC range). The anesthesiology protocol did not include hypotension management with fluid expansion or inotropes. Twenty minutes before the end of the intervention, all patients received Paracetamol $15 \mathrm{mg} / \mathrm{kg}$, as an analgesic. The analgesia was consolidated with surgical wound infiltration using levobupivacaine and lidocaine for both open and laparoscopic surgery.

\section{Statistical analysis}

Quantitative variables were described as the mean (SD) and compared among the different time intervals with population averaged mixed multilevel models to take into account the clustered nature of the data.

Probability values of less than 0.05 were considered statistically significant. All statistical analyses were performed using the SPSS statistical package (SPSS, Chicago IL, USA) and Stata 8.0.

\section{Results}

During the surgical procedure, a significant variation in $\mathrm{rScO}_{2}$ was observed at the different time points in comparison with basal values $(\mathrm{P}<0.01)$. In the LAP group, a significant $\mathrm{rScO}_{2}$ increase was noted at $\mathrm{T} 1$ $(\mathrm{P}<0.001)$. The intraoperative $\mathrm{rScO}_{2}$ values at T2 $(\mathrm{P}=0.6), \mathrm{T} 3(\mathrm{P}=0.4), \mathrm{T} 4(\mathrm{P}=0.5)$ and T5 (0.7) were not different compared with $\mathrm{T} 1$. The minimal $\mathrm{rScO}_{2}$ variations during the laparoscopic procedure were not related to $\mathrm{PP}$ pressure $(\mathrm{P}=0.5)$ or $\mathrm{CO}_{2}$ flow rate $(\mathrm{P}=0.8)$.

In the Open group a significant $\mathrm{rScO} 2$ increase was recorded at $\mathrm{T} 1(\mathrm{P}<0.001)$. At T3 $(\mathrm{P}=0.007)$ and $\mathrm{T} 5(\mathrm{P}=0.007) \mathrm{rScO} 2$ was significantly lower than at $\mathrm{T} 1$. The $\mathrm{rScO} 2$ profile and parameter mean values are reported in Figure 1A and Table 1.

\section{Heart rate}

HR showed significant changes during the entire surgical procedure in comparison with T0 $(\mathrm{P}=0.02)$. In the LAP group, HR was significantly higher at $\mathrm{T} 2$ compared with T0 $(\mathrm{P}=0.04)$. HR changes were related to $\mathrm{CO}_{2}$ PP pressure $(\mathrm{P}=0.002)$. An $\mathrm{HR}$ increase was also observed at T5 $(\mathrm{P}=0.009)$ compared with basal values.

In the Open group, HR changes at $\mathrm{T} 1$ $(\mathrm{P}=0.4)$, T3 $(\mathrm{P}=0.6)$ and $\mathrm{T} 5(\mathrm{P}=0.3)$ were not significantly different in comparison with T0. The HR profile and parameter mean values are given in Figure $1 \mathrm{~B}$ and Table 1.

\section{Peripheral oxygen saturation}

The $\mathrm{SatO}_{2}$ values were significantly different during the surgical intervention $(\mathrm{P}<0.001)$.

In the LAP group, a significant $\mathrm{rScO}_{2}$ increase was noted at $\mathrm{T} 1(\mathrm{P}=0.001)$. The $\mathrm{SatO}_{2}$ values at $\mathrm{T} 2$ were not significantly different in comparison with $\mathrm{T} 0(\mathrm{P}=0.3)$, but were lower than $\mathrm{T} 1(\mathrm{P}=0.06)$. The $\mathrm{SatO}_{2}$ differences between $\mathrm{T} 0$ and $\mathrm{T} 3$ and $\mathrm{T} 4$ were not significant $(\mathrm{P}=0.9$ and $\mathrm{P}=0.4$, respectively). A relevant decrease in $\mathrm{SatO}_{2}$ was observed at $\mathrm{T} 5$ in comparison with $\mathrm{T} 0$ $(\mathrm{P}<0.001)$. In the Open groups, $\mathrm{SatO}_{2}$ at $\mathrm{T} 1$ $(\mathrm{P}=0.27)$ and $\mathrm{T} 3(\mathrm{P}=0.34)$ was not different in comparison with T0. A significant decrease in $\mathrm{SatO}_{2}$ was recorded at $\mathrm{T} 5$ compared to $\mathrm{T} 0(\mathrm{P}<0.001)$. The $\mathrm{SatO}_{2}$ profile and parameter mean values are reported in Figure 1C and Table 1.

\section{Diastolic pressure}

The changes in DP values were significant during the surgical intervention $(\mathrm{P}<0.001)$. In the LAP group, a decrease in DP was significantly induced at $\mathrm{T} 1$ $(\mathrm{P}<0.001)$. At $\mathrm{T} 2$, even though the DP was lower than at $\mathrm{T} 0(\mathrm{P}=0.01)$, an increase in pressure was recorded compared with $\mathrm{T} 1$ $(\mathrm{p}<0.001)$; these changes were related to $\mathrm{CO}_{2} \mathrm{PP}(\mathrm{P}<0.001)$. During the surgical procedure, DP values remained significantly lower compared with $\mathrm{T} 0(\mathrm{P}<0.001)$, without a significant difference between $\mathrm{T} 4$ and $\mathrm{T} 1$ $(\mathrm{P}=0.13)$. A DP recovery was observed at T5 $(\mathrm{P}=0.05)$.

In the Open group, a significant decrease in DP was revealed at $\mathrm{T} 1$ $(\mathrm{P}<0.001)$. A progressive DP increase was recorded at $\mathrm{T} 3(\mathrm{P}=0.006)$ and $\mathrm{T} 5(\mathrm{P}=0.05)$. The DP profile and parameter mean values are given in Figure 1D and Table 1.

\section{Systolic pressure}

The SP values were significantly different during the surgical intervention $(\mathrm{P}<0.001)$. In the LAP group, a decrease in 
SP was significantly induced at $\mathrm{T} 1$ $(\mathrm{P}<0.001)$. At $\mathrm{T} 2$, an increase in pressure was recorded $(\mathrm{P}=0.15$ vs $\mathrm{T} 0 ; \mathrm{P}<0.001$ vs $\mathrm{T} 1)$; these changes were related to IAP $(\mathrm{P}<0.001)$. SP remained lower at $\mathrm{T} 3$ $(\mathrm{P}=0.006)$ and $\mathrm{T} 4(\mathrm{P}=0.004)$ compared with T0. At T4, the SP change was related to $\mathrm{CO}_{2}$ $\mathrm{PP}$ desufflation $(\mathrm{P}<0.001)$. At $\mathrm{T} 5$, no significant SP difference was noted in comparison with $\mathrm{T} 0(\mathrm{P}=0.6)$.

In the Open group, a significant decrease in SP was revealed at $\mathrm{T} 1(\mathrm{P}=0.01)$. A progressive systolic $\mathrm{PA}$ increase was recorded at $\mathrm{T} 3(\mathrm{P}=0.13)$ and $\mathrm{T} 5(\mathrm{P}=0.5)$. The SP profile and parameter mean values are given in Figure 1E and Table 1.

\section{End-tidal $\mathrm{CO}_{2}$}

During the surgical procedure, no significant variations in End-tidal $\mathrm{CO}_{2}$ were observed at the different time points in comparison with $\mathrm{T} 1 \quad(\mathrm{P}=0.37)$. The End tidal mean values are provided in Table 1 . In Table 1, the hemodynamic mean values at the different surgical time points are reported.

\section{Discussion}

During laparoscopic procedures in children, adaptive changes in cerebral and systemic oxygenation and hemodynamic parameters are observed. These changes are related to pathophysiological consequences induced by PP creation and the impact of general anesthesia. Adverse hemodynamic events may be prevented with stringent intraoperative technical monitoring and strict standardization of the pediatric anesthesiological protocol. As in adults, pediatric laparoscopy is less invasive in nature, thereby providing a more rapid recovery, shorter hospital stay, decreased postoperative pain and improved cosmetic outcome when compared with traditional open surgery. Nevertheless, the procedure may be associated with hemodynamic alterations generated by the high intra-abdominal pressure brought on by PP creation and by the existence of insufflation gas that is absorbed by the blood. These systemic hemodynamic alterations may result in changes in endorgan blood flow and oxygen delivery. In addition, the impact of anesthesia on hemodynamic changes during laparoscopy should be considered.15,18-21 To date, data regarding hemodynamic changes during laparoscopy are conflicting and further evaluations in very small children represent new research perspectives for the near future. The most commonly studied parameters are HR, systemic vascular resistance, mean arterial pressure and central venous pressure. Increases and decreases in virtually all of the parameters noted above have been described after the institution of PP. The data vary in relation to the many factors affecting these values such as the child's weight and age. 22

Significant changes in cerebral oxygenation occur in some patients during $\mathrm{CO}_{2}$ insufflation. To date, the data collected regarding alterations in cerebral oxygenation during laparoscopic procedures in children have been limited and non-homogenous. ${ }^{11-15}$

Previously, we reported on changes in cerebral oxygenation during laparoscopic procedures in pediatric patients, when strin- gent monitoring was not adopted. ${ }^{15}$ In this study, we closely monitored cerebral oxygenation with NIRS and the anesthesiologists were not blinded to the readings to prevent intraoperative alterations. Under these conditions, we showed that $\mathrm{CO}_{2}$ insufflation during PP may not influence cerebral oxygenation during laparoscopic surgery. These results suggest that the impact of anesthesia on adaptive changes should not be underestimated and confirm that this technology may be useful to anticipate any potential decrease in brain oxygenation. Rapid recognition of low cerebral perfusion and prompt correction is a challenge and the main goal is to avoid negative postoperative neurodevelopmental outcomes following pediatric

Table 1. Hemodynamic mean values at the different surgical time points.

\begin{tabular}{|c|c|c|}
\hline Parameters & LAP group & Open group \\
\hline Operative time $($ mean $\pm \mathrm{SD})$ & $102 \pm 37.2$ & $71 \pm 36.57$ \\
\hline $\begin{array}{r}\text { NIRS } \\
\text { T0 } \\
\text { T1 } \\
\text { T2 } \\
\text { T3 } \\
\text { T4 } \\
\text { T5 } \\
\end{array}$ & $\begin{array}{c}73.8 \pm 5.7 \\
81.3 \pm 9.6 \\
82.0 \pm 11.1 \\
82.4 \pm 10 \\
82.1 \pm 9.7 \\
81.7 \pm 8.4\end{array}$ & $\begin{array}{c}74.9 \pm 9.0 \\
82.4 \pm 6.8 \\
- \\
77.1 \pm 9.2 \\
- \\
77.2 \pm 7.5\end{array}$ \\
\hline $\begin{array}{l}\text { Heart rate } \\
\text { T0 } \\
\text { T1 } \\
\text { T2 } \\
\text { T3 } \\
\text { T4 } \\
\text { T5 }\end{array}$ & $\begin{array}{c}99.5 \pm 22.9 \\
100.3 \pm 21.6 \\
107.3 \pm 25.2 \\
103.5 \pm 24.1 \\
105.7 \pm 23.0 \\
106.1 \pm 20.3\end{array}$ & $\begin{array}{c}115.3 \pm 11.1 \\
111.4 \pm 20.3 \\
- \\
113.1 \pm 18.3 \\
- \\
120.2 \pm 24.3\end{array}$ \\
\hline $\begin{array}{c}\mathrm{SatO}_{2} \\
\mathrm{~T} 0 \\
\mathrm{~T} 1 \\
\mathrm{~T} 2 \\
\mathrm{~T} 3 \\
\mathrm{~T} 4 \\
\mathrm{~T} 5 \\
\end{array}$ & $\begin{array}{l}98.2 \pm 1.0 \\
98.6 \pm 1.0 \\
97.9 \pm 0.9 \\
98.3 \pm 1.2 \\
97.9 \pm 0.1 \\
96.9 \pm 2.5\end{array}$ & $\begin{array}{c}98.6 \pm 1 \\
99.0 \pm 1 \\
- \\
99.0 \pm 1.3 \\
- \\
97.4 \pm 1.9\end{array}$ \\
\hline $\begin{array}{l}\text { Diastolic PA } \\
\text { T0 } \\
\text { T1 } \\
\text { T2 } \\
\text { T3 } \\
\text { T4 } \\
\text { T5 }\end{array}$ & $\begin{array}{c}63.1 \pm 15.1 \\
47.3 \pm 7.6 \\
56.4 \pm 11.6 \\
50.7 \pm 9.3 \\
50.8 \pm 9.3 \\
57.6 \pm 11.0\end{array}$ & $\begin{array}{c}58.2 \pm 17.3 \\
46.6 \pm 7.2 \\
51.2 \pm 10.3 \\
51.2 \pm 10.2 \\
- \\
55.5 \pm 10.3\end{array}$ \\
\hline $\begin{array}{c}\text { Systolic PA } \\
\text { T0 } \\
\text { T1 } \\
\text { T2 } \\
\text { T3 } \\
\text { T4 } \\
\text { T5 }\end{array}$ & $\begin{array}{c}111.1 \pm 17.4 \\
94.2 \pm 6.8 \\
107.5 \pm 15.2 \\
103.8 \pm 12.7 \\
103.4 \pm 13.0 \\
110.8 \pm 13.7\end{array}$ & $\begin{array}{c}102.9 \pm 8.5 \\
95.0 \pm 9 \\
- \\
101.7 \pm 11.9 \\
- \\
105.3 \pm 13.5\end{array}$ \\
\hline $\begin{array}{l}\text { End-tidal } \mathrm{CO}_{2} \\
\text { T0 } \\
\text { T1 } \\
\text { T2 } \\
\text { T3 } \\
\text { T4 } \\
\text { T5 } \\
\end{array}$ & $\begin{array}{c}- \\
35.8 \pm 5.8 \\
36.7 \pm 5.3 \\
38.3 \pm 4.8 \\
37.8 \pm 4.8 \\
-\end{array}$ & $\begin{array}{c}- \\
41.9 \pm 6.7 \\
- \\
37.5 \pm 6.3 \\
- \\
-\end{array}$ \\
\hline
\end{tabular}


surgical procedures. The anesthesiologist should be specifically prepared to manage this vulnerable population. ${ }^{23,24}$

In this study we also collected data on the impact of $\mathrm{CO}_{2}$ insufflation on peripheral oxygenation. Anesthesia induction and intraoperative management are critical determinants in peripheral oxygenation stability during laparoscopic procedures; in fact, during the latter part of surgery (last ten minutes), especially in very young children (whose muscles are very weak and do not intrude with surgical activity), a gradual reduction in ventilator assistance was adopted in order to facilitate gradual recovery of spontaneous breathing. 25,26

According to the literature, increases in arterial pressure during peritoneal insufflation have been noted. Moreover, we showed that a significant decrease in diastolic and systolic pressures, occurred at induction of anesthesia and during intraoperative surgical time points.

The pressure profile was similar in both open surgery and the laparoscopic surgical approach; this observation supports the hypothesis that PP creation is only partially responsible for pressure variations and underlines the importance of dedicated anesthesiological management in pediatrics even for traditional surgery.

As previously reported, ${ }^{15}$ during PP creation, an HR increase was recorded. The correlation between $\mathrm{HR}$ changes and $\mathrm{CO}_{2}$ flow rate support the role of a neurohumoral $\mathrm{CO}_{2}$ effect. $\mathrm{CO}_{2}$ stimulates the sympathoadrenal system causing a significant release of catecholamines and cortisol and thus increased HR. The absorption of $\mathrm{CO}_{2}$ during positive pressure pneumoperitoneum may lead to an increased $\mathrm{CO}_{2}$ load. While a different $\mathrm{CO}_{2}$ reabsorption in infants and children has already been reported based on the different characteristics in their peritoneal surface, $\mathrm{CO}_{2}$ elimination is related to age with younger children eliminating more $\mathrm{CO}_{2}$ than older children. ${ }^{27}$

In most patients undergoing controlled ventilation, end-tidal $\mathrm{CO}_{2}$ closely reflects arterial $\mathrm{CO}_{2}$ tension. ${ }^{27}$ We did not measure arterial $\mathrm{CO}_{2}$ and arterial $\mathrm{pH}$ levels in this study, but we assume that because there were only minor changes in end-tidal $\mathrm{CO}_{2}$, that the blood gases did not change significantly. Clinically, we did not observe any adverse cardiovascular or respiratory events in our patients.

The present preliminary report has several limitations. The sample size was limited and a study on a larger number of chil-

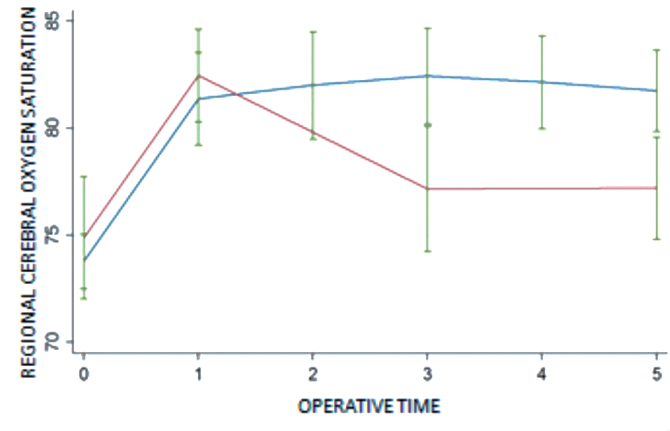

A

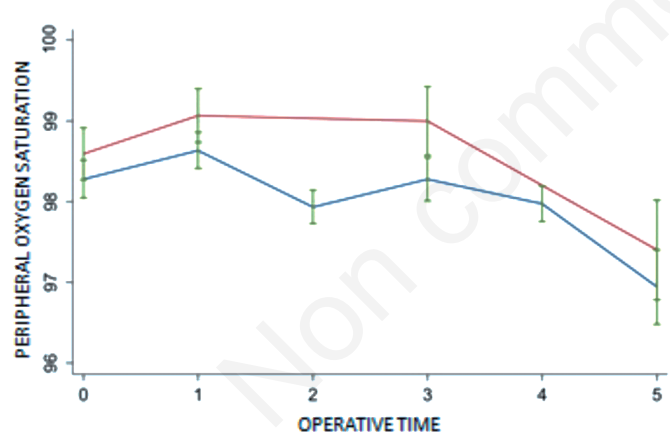

C

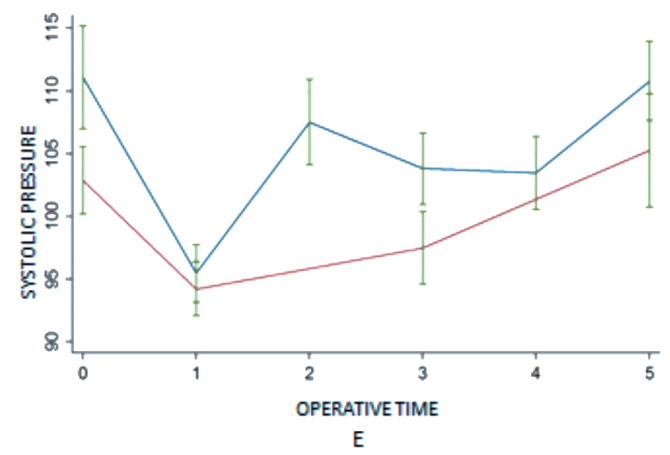

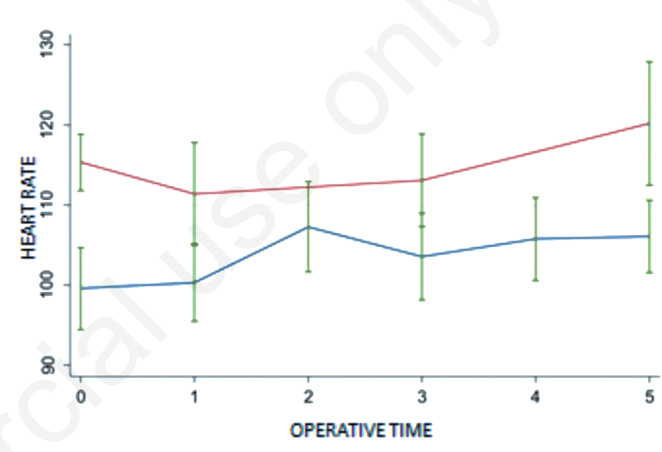

B

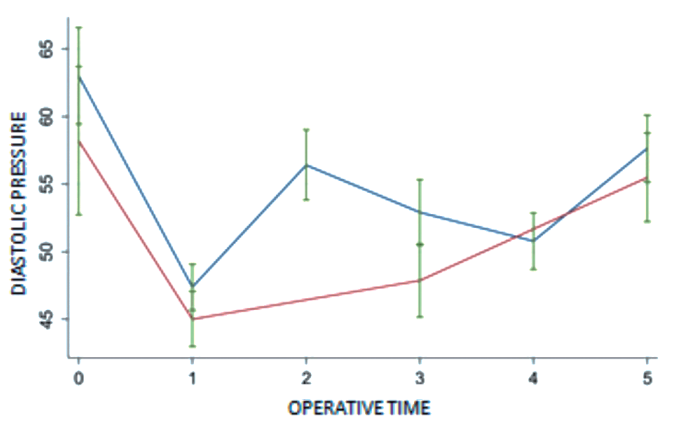

D

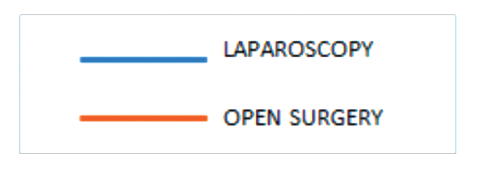

Figure 1. Profile of regional cerebral oxygen saturation (A), heart rate (B), peripheral oxygen saturation (C), diastolic pressure (D) and systolic pressure (E) in LAP and OPEN groups at the different surgical time points. 
dren is mandatory to confirm the results. Secondly, it concerns a population with a broad range of ages, from 1 to 18 years, and the impact of IAP, absorption and elimination of $\mathrm{CO}_{2}$ and anesthesia protocols are different for infants, children and adolescents. The initial inclusion criteria for recruitment in the study selected an homogenous group of procedures, however the influence of the different operative times on the $\mathrm{rScO} 2$ cannot be excluded. The anesthesiologist's anesthesia technique and ventilation strategy to obtain the best parameters were not standardized. Additionally, the exact impact of the anesthesia on hemodynamics could be supported by hemodynamic evaluation during the standard anesthesia protocol and using different anesthesiological agents. Finally, arterial blood gas analysis monitoring would be useful during laparoscopic procedures; in our study, the routine procedure, did not call for invasive $\mathrm{PaCO}_{2}$ monitoring.

This study points to new study directions to ameliorate our knowledge on the combined surgical and anesthesiological role on hemodynamic status in pediatric laparoscopy. With the advent of new mini invasive surgical approaches, there is a need for specific and dedicated anesthesiological procedures, particularly in children. Although anesthesia for laparoscopic surgery does not require a major extension of the traditional methods for pediatric abdominal surgery, special consideration must be given to alterations in cardiovascular and respiratory status that occur during the laparoscopic procedure. ${ }^{18-21}$ Children, infants and neonates represent an anesthesiological challenge because of age-specific anatomical and physiological issues. Apart from these pediatric-specific considerations, the pediatric anesthesiologist must understand the implications of laparoscopic surgery, and prevent or act accordingly to changes that will occur during these procedures. 18-21 There is room for improvement in quality of care during the laparoscopic surgical approach in pediatrics.

\section{Conclusions}

This study demonstrates that pathophysiological hemodynamic alterations are influenced by both the procedure and the anesthesia during pediatric abdominal laparoscopic surgery. However, the exact role of each factor needs further research. Due to age-related homeostatic vulnerability, hemodynamic status should not be underestimated in pediatrics. Knowledge of the pathophysiological changes, the stan- dardization of intraoperative surgical assessment and anesthesia management are all mandatory to prevent adverse hemodynamic outcomes. Future challenges will include appropriate application of combined minimally invasive surgery and anesthesiological protocols, while maintaining the child's safety during and after laparoscopic procedures.

\section{References}

1. Maesani M, Pares F, Michelet D, et al. Haemodynamic and cerebral oxygenation during paediatric laparoscopy in fluid optimized patients. Br J Anaesth 2016;116:564-6.

2. de Waal EE, de Vries JW, Kruitwagen $\mathrm{CL}$, et al. The effects of low-pressure carbon dioxide pneumoperitoneum on cerebral oxygenation and cerebral blood volume in children. Anesth Analg 2002;94:500-5.

3. Schauer PR, Schwesinger WH. Hemodynamic effects of laparoscopy. Surg Endosc 1995;9:119-20.

4. Gupta R, Singh S. Challenges in paediatric laparoscopic surgeries. Indian $\mathrm{J}$ Anaesth 2009;53:560-6.

5. Tam PK. Laparoscopic surgery in children. Arch Dis Child 2000;82:240-3.

6. Hodgson C, McClelland R, Newton J. Some effects of the peritoneal insufflation of carbon dioxide at laparoscopy. Anaesthesia 1970;25:382-90.

7. Joris JL, Hinque VL, Laurent PE, et al. Pulmonary function and pain after gastroplasty performed via laparotomy or laparoscopy in morbidly obese patients. Br J Anaesth 1998;80:283-8.

8. Joris JL, Chiche JD, Canivet JL, et al. Hemodynamic changes induced by laparoscopy and their endocrine correlates: effects of clonidine. J Am Coll Cardiol 1998;32:1389-96.

9. Wahba RW, Béique F, Kleiman SJ. Cardiopulmonary function and laparoscopic cholecystectomy. Can J Anaesth. 1995;42:51-63.

10. Tuna AT, Akkoyun I, Darcin S, et al. Effects of carbon dioxide insufflation on regional cerebral oxygenation during laparoscopic surgery in children: a prospective study. Braz J Anesthesiol 2016;66:249-53

11. Tsypin LE, Mikhel'son VA, Chusov KP, et al. Central and cerebral hemodynamics during gynecological laparoscopic interventions in children. Anesteziol Reanimatol 2007;1:30-2.

12. Tytgat SH, Stolwijk LJ, Keunen K, et al. Brain oxygenation during laparoscopic correction of hypertrophic pyloric stenosis. J Laparoendosc Adv Surg Tech 2015;A25:352-7.

13. Tytgat SH, van Herwaarden MY, Stolwijk LJ, et al. Neonatal brain oxygenation during thoracoscopic correction of esophageal atresia. Surg Endosc Surg Endosc 2016;30:2811-7.

14. Bishay M, Giacomello L, Retrosi G, et al. Decreased cerebral oxygen saturation during thoracoscopic repair of congenital diaphragmatic hernia and esophageal atresia in infants. J Pediatr Surg 2011;46:47-51.

15. Pelizzo G, Bernardi L, Carlini V, et al. Laparoscopy in children and its impact on brain oxygenation during routine inguinal hernia repair. J Minim Access Surg 2017;13:51-6.

16. Jackson HT, Kane TD. Advances in minimally invasive surgery in pediatric patients. Adv Pediatr 2014;61:149-95.

17. Truchon R. Anaesthetic considerations for laparoscopic surgery in neonates and infants: a practical review. Best Pract Res Clin Anaesthesiol 2004;18:343-55.

18. Lasersohn L. Anaesthetic considerations for paediatric laparoscopy. S Afr J Surg 2011;49:22-6.

19. Srivastava A, Niranjan A. Secrets of safe laparoscopic surgery: Anaesthetic and surgical considerations. J Minim Access Surg 2010;6:91-4.

20. Ahmed A. Laparoscopic surgery in children: anaesthetic considerations. J Pak Med Assoc 2006;56:75-9.

21. Ahmed M, Nessa M, Islam MS, et al. Effects of pneumoperitoneum during laparoscopic surgery in young children. JAFCM Bangledesh 2009;5:18-20.

22. Hardacre JM, Talamini MA. Pulmonary and hemodynamic changes during laparoscopy: are they important? Surgery 2000;127:241-4.

23. Rhondali O, Pouyau A, Mahr A, et al. Sevoflurane anesthesia and brain perfusion. Paediatr Anaesth 2015;25:180-5.

24. Rhondali O, Juhel S, Mathews S, et al. Impact of sevoflurane anesthesia on brain oxygenation in children younger than 2 years. Paediatr Anaesth 2014;24:734-40.

25. Goligher EC, Ferguson ND, Brochard LJ. Clinical challenges in mechanical ventilation. Lancet. 2016;387:1856-66.

26. El-Khatib MF, Bou-Khalil P. Clinical review: liberation from mechanical ventilation. Crit Care 2008;12:221.

27. McHoney M, Corizia L, Eaton S, et al. Carbon dioxide elimination during laparoscopy in children is age dependent. J Pediatr Surg 2003;38:105-10. 